\title{
A novel measurement-based approach for modeling and computing interference factors for wireless channels
}

\author{
Alper Rifat Ulucinar ${ }^{1 *}$, Ibrahim Korpeoglu and Ezhan Karasan ${ }^{2}$
}

\begin{abstract}
Wireless communication technologies divide their available spectrum into pre-defined channels. Some wireless technologies, such as the IEEE $802.11 \mathrm{~b} / \mathrm{g}$, define their channels in such a way that adjacent channels share the spectrum. When two distinct channels share some part of their spectrum, simultaneous transmissions on these channels cause what is known as the adjacent channel interference. For problems that consider adjacent channel interference, such as the channel assignment problem for multi-channel wireless mesh networks, we need a model that quantitatively describes adjacent channel interference. The interference factor is a concept defined to quantify the amount of the overlap, hence the interference, between two wireless channels. Analytical and experimental methods have been proposed in the literature to define the interference factors among channels of a wireless technology. In this article, we propose a physical-layer-measurement-based, technology-independent and generic approach that is capable of determining interference factors between the channels of a wireless technology and also between the channels of two different wireless technologies, such as IEEE 802.11 and IEEE 802.15.4. We also report our measurement results for interference factors among 802.11 b DSS channels and between 802.15.4 and 802.11b channels. Our results show that our approach is practical, accurate and generic enough to compute the interference factors of radio channels belonging to various wireless communication technologies.
\end{abstract}

Keywords: Radio channels, Overlapping and orthogonal channels, Interference factor, 802.11, 802.15.4, Spectrum analyzer

\section{Introduction}

Interference factor (I-factor) [1] is used to model the amount of interference between two channels of a wireless communication standard, such as the IEEE 802.11 family of standards or the IEEE 802.15.4 standard. Spectral bands are allocated to wireless communication standards by regulation and standardization bodies such as the US Federal Communications Commission (FCC) or the European Telecommunications Standards Institute (ETSI). These standards further divide the band allocated to them into channels. Each standard has its own channel definition, some of which are compatible and some of which are not. For instance, the IEEE 802.11g standard is compatible with

\footnotetext{
*Correspondence: ulucinar@cs.bilkent.edu.tr

${ }^{1}$ Department of Computer Engineering, Bilkent University, 06800, Ankara, Turkey

Full list of author information is available at the end of the article
}

the $2.4 \mathrm{GHz}$ DSSS channel definitions of the $802.11 \mathrm{~b}$ standard for supporting legacy devices, whereas the channel definitions of the $802.11 \mathrm{~g}$ and IEEE 802.15.4 standards are not compatible with each other. Furthermore, a standard may define more than one channel structure if it employs multiple PHY service specifications. As an example, the IEEE 802.11 standard defines different channel structures for the frequency-hopping spread spectrum (FHSS) PHY and the direct-sequence spread spectrum (DSSS) PHY services [2].

The definition of the channels of a wireless communication standard comprises the center frequencies and the bandwidths of the channels. For instance, the IEEE $802.11 \mathrm{~b}$ and IEEE $802.11 \mathrm{~g}$ standards use the same channel structure for the DSSS PHY. The $802.11 \mathrm{~b} / \mathrm{g}$ DSSS channels are in the $2.4 \mathrm{GHz}$ industrial, scientific and medical (ISM) band. The center frequency of the first $802.11 \mathrm{~b} / \mathrm{g}$ DSSS

\section{是 Springer}

(c) 2013 Ulucinar et al: licensee Springer. This is an Open Access article distributed under the terms of the Creative Commons Attribution License (http://creativecommons.org/licenses/by/2.0), which permits unrestricted use, distribution, and reproduction in any medium, provided the original work is properly cited. 
channel (channel id 1) is $2412 \mathrm{MHz}$. The center frequencies of consecutive channels are separated by $5 \mathrm{MHz}$ and the bandwidth of each channel is $22 \mathrm{MHz}$. The standard defines 14 channels, of which the first 11 are supported in the FCC domain.

For two radios to communicate with each other using a communication standard, they must be tuned to the same channel, which can be shared by other nearby transmitters. The 802.11 medium access control (MAC) sublayer coordinates transmissions on the same channel using the Carrier Sense Multiple Access/Collision Avoidance (CSMA/CA) protocol. A medium-access control protocol is needed for, among other functions, controlling and scheduling transmissions of contending radios. Two radios are said to be contending for the channel if they simultaneously have packets to send through the wireless medium. However, the distributed stochastic operation of the 802.11 MAC sometimes fails to properly schedule the contenders, so more than one transmitters concurrently attempt to send packets. This causes simultaneous transmissions from multiple radios that interfere with each other.

To further complicate the situation, the interfering radio might be operating on a different (but adjacent or overlapping) channel as the channel the receiver under discussion is using. Some wireless communication standards (for example, IEEE 802.11a) define their channel structures in such a way that it is impossible for a radio to receive any signal power from a transmitter not tuned to the same channel as itself. However, for other standards (such as the $802.11 \mathrm{~b} / \mathrm{g}$ DSSS PHY), it is possible for a receiver operating on channel $i$ to receive interference power from a transmitter operating on one of the channels $i, i \pm 1, i \pm$ $2, i \pm 3$, or $i \pm 4$. This occurs because of how DSSS channels as well as the transmit spectrum mask (TSM) are defined in $802.11 \mathrm{~b} / \mathrm{g}$. The DSSS TSM [2] allows a transmission bandwidth of $22 \mathrm{MHz}$ centered around the channel center frequency. Therefore, two transmitters tuned to channels $i$ and $j$ share the wireless medium (a common frequency range) as long as $i$ and $j$ 's center frequencies are separated by less than $22 \mathrm{MHz}$. Because the center frequencies of consecutive channels are $5 \mathrm{MHz}$ apart from each other, this translates into a channel separation, $|i-j|$, of fewer than $\lceil 22 / 5\rceil=5$ channels.

The term overlapping channels [1] (or non-orthogonal channels) is used to describe a relation between at least two channels that share a frequency range. When we consider the 802.11 DSSS PHY specification, two channels $i$ and $j$ are overlapping channels if and only if $|i-j|<5$. If $|i-j| \geq 5$, channels $i$ and $j$ are called non-overlapping or orthogonal channels.

The interference factor quantitatively defines the amount of overlap and interference between two wireless channels. In this article, we propose a novel and practical approach that can be used to compute the interference factor values between not only the channels of a single standard but also between the channels of two different standards sharing the same spectrum. Our approach is based on physical-layer measurements and has the advantage of being practically applicable to various wireless communication standards. Existing analytical or measurement-based approaches lack this important property because of their dependence on a specific wireless standard. The main contributions of our article are as follows:

- To the best of authors' knowledge, the physical-layer-measurement-based methods we propose are the first in the literature that are generic enough to model the interference between channels of any two wireless communication technologies, such as 802.11 and 802.15.4. They can also be used to obtain the I-factor values between channels of the same wireless technology, such as 802.11 channels.

- These methods are also capable of modeling interference on wireless communication devices caused by non-communication devices such as microwave ovens.

- Using the proposed methods and a $2.4 \mathrm{GHz}$ spectrum analyzer, we give our measurement results for the proposed I-factors between 802.11b DSSS channels and we compare our results with those of other analytical and measurement-based I-factor models in the literature. We also report our measurement results on the interference from an 802.15.4 (ZigBee) transmitter on an $802.11 \mathrm{~b}$ receiver.

The remainder of the article is organized as follows: In Section 2, we introduce the concept of the I-factor in more detail. In Section 3, we discuss some related study on Ifactor modeling. In Section 4, we present and discuss our novel methods. In Section 5, we report our measurement results for the I-factors between 802.11b DSSS channels and between 802.15 .4 and $802.11 \mathrm{~b}$ channels. Finally, in Section 6, we conclude the article.

\section{Interference factor}

The concept of an interference factor [1] is used as a model of how much interference power will leak from adjacent channels. An I-factor is commonly defined as a value in the interval $[0,1]$, where 0 means no interference, and 1 means maximum interference (when the interferer's frequency band intersects maximally with the transmitter's frequency band). Ideally, a transmitter operating on a nonoverlapping channel with respect to a receiver generates no interference on the receiver, therefore the I-factor for two non-overlapping channels is 0 . When the interferer and receiver radios are operating on the same channel, $100 \%$ of the interferer signal power at the location of the 
receiver will pass through the receiver's filter. Hence, the I-factor is defined as 1 for two channels $i$ and $j$ if $i=j$.

For overlapping channels, an intuitive definition of the Ifactor is given in $[1,3]$ as follows: If $P_{i}$ is the received power of a particular signal (sent by a transmitter on channel $j$ ) at a particular location by a receiver tuned to channel $i$, and $P_{j}$ is the received power of the same signal at the same location by a receiver tuned to channel $j$, then the I-factor of channel $j$ on channel $i$ is defined as:

$$
I(i, j)=\frac{P_{i}}{P_{j}} .
$$

Two important properties of the I-factor definition given above are: (a) it is not a commutative operation, i.e., $I(i, j)$ is not necessarily equal to $I(j, i)$, and (b) $I(i, j) \in[0,1]$ (assuming the transmitter is transmitting on channel $j$ ).

It may also be possible to define the I-factor analytically. One such approach for the interference factor of 802.11 DSSS channels appears in [1]. According to this model, the I-factor for two (overlapping) 802.11 channels is calculated as a function of the power spectral distribution of the DSSS signal and the receiver filter's frequency response, as follows:

$$
\begin{aligned}
I_{\text {theory }}(i, j) & =\operatorname{IF}_{(T, R)}(5|i-j|) \\
& =\int_{-\infty}^{\infty} P_{\mathrm{DSSS}}\left(f, F_{c, t}\right) B_{R}\left(f, F_{c, t}-5|i-j|\right) d f
\end{aligned}
$$

where $P_{\text {DSSS }}\left(f, F_{c, t}\right)$ is the power spectral distribution function for the interferer DSSS signal, with a center frequency of $F_{c, t}$ (in $\mathrm{MHz}$ ), and $B_{R}\left(f, F_{c, r}\right)$ is the receiver bandpass filter's frequency response, with $F_{c, r}=F_{c, t}-$ $5|i-j|$ (in $\mathrm{MHz}$ ). In this idealized discrete model, the transmitted signal's power distribution $\left(P_{\mathrm{DSSS}}\left(f, F_{c, t}\right)\right)$ and the receiver filter $\left(B_{R}\left(f, F_{c, r}\right)\right)$ are approximated with the DSSS TSM defined by the standard, with center frequencies of $F_{c, t}$ and $F_{c, r}$, respectively [1].

The first I-factor definition we propose in this article is the ratio of the area below the intersection of the interferer and the receiver channel signal traces on a spectrum analyzer to the total area below the interferer's signal trace, and is expressed mathematically as follows for interferer channel $i$ and receiver channel $j$ :

$$
I(i, j)=\frac{\int_{f_{l}}^{f_{u}} \min \left\{P_{\text {intf }}\left(f, F_{c, i}\right), P_{\text {recv_ch }}\left(f, F_{c, j}\right)\right\} d f}{\int_{f_{l}}^{f_{u}} P_{\text {intf }}\left(f, F_{c, i}\right) d f}
$$

where $P_{\text {intf }}\left(f, F_{c, i}\right)$ is the interferer signal's power spectral distribution trace captured with a spectrum analyzer, and $P_{\text {recv_ch }}\left(f, F_{c, j}\right)$ is the power spectral distribution trace captured with a spectrum analyzer that belongs to a transmitted signal using the same standard as the receiver radio and that is on the same channel as the receiver (channel $j$ ).
$F_{c, i}$ is the center frequency of the interferer signal's channel, which is specific to the standard the interferer radio belongs to. Similarly, $F_{c, j}$ is the center frequency of the receiver's channel, which is specific to the standard the receiver radio belongs to. The interferer and the receiver radios might belong to different wireless communication standards as well as to the same standard. The unit of power used in the calculation of $I(i, j)$ in $(3)$ is the unit used in the spectrum analyzer's traces. The lower and upper limits of the integrations $\left(f_{l}\right.$ and $f_{u}$, respectively) are determined by the total spectrum band covered by the traces of the interferer and the receiver channel signals. We call this method the signal intersection area method (SIAM) in this article and detail it in Section 4.

The second I-factor definition we propose for quantifying interference between the interferer's channel $i$ and the receiver's channel $j$ is the ratio of the total received interference energy on channel $j$ radiated from a transmitter on channel $i$ to the total energy received from the same transmitter by a receiver on channel $i$. Equation (4) expresses this ratio in mathematical terms:

$$
I(i, j)=\frac{\int_{f_{l}}^{f_{u}} P_{\text {intf }}\left(f, F_{c, i}\right) B_{R}\left(f, F_{c, j}\right) d f}{\int_{f_{l}+\left(F_{c, i}-F_{c, j}\right)}^{f_{u}+\left(F_{c, i}-F_{c, j}\right)} P_{\text {int }}\left(f, F_{c, i}\right) B_{R}\left(f, F_{c, i}\right) d f},
$$

where the definitions of $P_{\text {intf }}\left(f, F_{c, i}\right), F_{c, i}$, and $F_{c, j}$ are as in (3). $B_{R}$ is the receiver bandpass filter's frequency response. In (4), we use this response twice, once for a receiver tuned to the channel with the center frequency, $F_{c, j}$, and once for another receiver tuned to the same channel as the interferer itself, $F_{c, i}$. We obtain $P_{\text {intf }}\left(f, F_{c, i}\right)$ experimentally using a spectrum analyzer. The lower limit of integration, $f_{l}$, for the receiver channel $j$ is $f_{l}=F_{c, j}-\frac{B w}{2}$, and the upper limit of integration, $f_{u}$, for the receiver channel $j$ is $f_{u}=F_{c, j}+\frac{B w}{2}$, where $B w$ is the width of the bandpass filter's response. This method, which we call percentage of maximum interference energy (PMIE), has a more concrete physical interpretation that we discuss in detail in Section 4.

\section{Related study}

There are two main classes of I-factor models in the literature. The first class comprises analytical models [1], which are generally applied to relatively simple modulation techniques, such as the DSSS, because of the complexities of the models. The second class comprises a set of experimental measurement-based methods [3], which are more flexible than the analytical methods because they are not built upon the specifics of a physical-layer technique; they involve measurements in any of the various layers of the open systems interconnection (OSI) stack [4]. The most direct and more commonly adopted experimental method of obtaining an I-factor model is to perform signal-tonoise ratio (SNR) [5] measurements. In these models, a 
receiver is kept fixed at a channel and its transmitter is operated on non-overlapping and overlapping channels. For each channel of the transmitter, SNR is measured on the receiver and normalized to a scale of $[0,1]$ as in [3]. This method mandates that the interferer (transmitter) and the receiver must be using the same wireless communication standard, so that SNR readings (where the signal belongs to the interferer) are available at the receiver. If the interferer uses a different wireless communication standard than the receiver (such as the interferer being a Bluetooth radio and the receiver being an $802.11 \mathrm{~b} / \mathrm{g}$ radio), then there will be no links between these two radios and no SNR measurements will be available at the receiver radio.

In [6,7], Feng and Yang use numerical methods to analyze network capacity improvements that can be gained by using partially overlapping channels. While defining the carrier sensing range between two nodes operating on channels $i$ and $j$, they perform a set of testbed experiments that involve two pairs of nodes. One pair communicates with each other on channel $i$ and the other pair communicates on channel $j$. The authors define the carrier sensing range as "the maximum distance that these two can affect each other's communications" [7]. Then they give statistical and numerical models of capacity improvements when overlapping channels are used compared to using only orthogonal channels in one-hop and multi-hop wireless networks. In [7], the authors also discuss the cases where no improvement can be gained by using partially overlapping channels.

In [8], Zhou et al. envision that in the very near future, the world will be full of low-power wireless sensors sharing the same spectrum. As an illustrative example, they measure the $2.4 \mathrm{GHz}$ spectrum with their HP 8593E spectrum analyzer in the coexistence of a microwave oven, a cordless $2.4 \mathrm{GHz}$ presenter, and a MICAz sensor network. They also report the reception ratios of the MICAz motes when the microwave oven is on and when it is off. However, they do not model interference using these measurements. The authors propose the dimensions along which new wireless sensor network protocols should be designed to cope with the crowded spectrum issue.

In [9], Fuxjäger et al. pose the fundamental question of whether there really is no interference between the nonoverlapping channels of IEEE 802.11. To investigate this, the authors use a testbed consisting of four laptops, each equipped with an Intel PRO 2200BG mini-pci card and running Linux. They place the laptops on a linear line-ofsight topology, each raised $1.5 \mathrm{~m}$ above the ground. Using this testbed, the authors measure the MAC and transport layer throughputs and MAC frame loss ratios. They also measure the goodput of a TCP flow. The authors conclude that due to the near-far effect [10], cross-channel interference exists between non-overlapping channels of
IEEE 802.11 when the receiver and the interferer radios are placed only tens of centimeters away from each other. They also conclude that off-the-shelf IEEE 802.11 chipsets may not be ready to be placed in the same box for use in multi-radio wireless mesh networks.

In [11], Petrova et al. investigate the performance of IEEE 802.15.4 networks under the interference caused by IEEE 802.11g and pre-standard IEEE 802.11n networks through measurements. They use a testbed consisting of an $802.11 \mathrm{~g} / \mathrm{n}$ access point, a laptop used as the $802.11 \mathrm{~g} / \mathrm{n}$ traffic sink and equipped with an $802.11 \mathrm{~g} / \mathrm{n}$ adapter, a PC used as the $802.11 \mathrm{~g} / \mathrm{n}$ traffic generator, and two TelosB motes. They also monitor the $2.4 \mathrm{GHz}$ spectrum with an Agilent E4440A spectrum analyzer. Using this testbed, the authors measure the packet delivery ratios of the 802.15.4 network. They use the spectrum analyzer to report the average power spectral densities of the $802.11 \mathrm{n}$ signals for different alignments of the 802.11 n nodes. However, they do not model interference using these measurements.

\section{Our proposed interference factor calculation methods}

The method we follow to compute the I-factor is based on physical-layer measurements in the frequency domain, taken with a spectrum analyzer. Using the spectrum analyzer, we obtain signal traces showing the power spectral distribution of a transmitted wireless signal. To capture channel activity, we generate and analyze $802.11 \mathrm{~b} / \mathrm{g}$ packets using the multi-radio mesh nodes in our testbed BilMesh, which is a multi-hop, multi-radio wireless mesh networking testbed we have established at Bilkent University. To analyze the 802.15 .4 interference on 802.11 receivers, we add ZigBee motes to our testbed. We use a Yellowjacket-Tablet 2.4/5 GHz Wi-Fi spectrum analyzer [12] to obtain the signal traces. The setup required for the measurements consists of a single wireless transmitter. However, as explained in Section 5.3, two or more transmitters operating on the same channel can be used to shorten the time needed to collect the signal traces. The transmitter(s') channel is set in succession to one of the channels of interest between which the I-factor is to be calculated.

After collecting the traces for the receiver and interferer channel signals, we consider the area below the intersection of these traces in relation to the power spectral density of the interferer signal. The ratio is considered to be the I-factor between two channels. This I-factor definition has been introduced formally in Section 2 as SIAM. Below, we describe the steps showing how it can be computed:

1. We obtain the spectrum analyzer traces for the two wireless signals between which the I-factor is to be calculated. Because I-factor is defined as the ratio of 
the received energy on a receiver channel to the radiated energy on a transmitter (interferer) channel, we have to obtain the interferer's power spectral distribution using the spectrum analyzer. In our current implementation, we obtain, store, and process this information with device independent bitmap files. We use bitmap files because the spectrum analyzer available to us can export this data in bitmap format; however any other form of representation for the power spectral density data can be used with our method.

2. Once the two signal traces are obtained, the area of their intersection in the frequency domain over a reference power level is calculated. In our current implementation, the reference power level is determined by the user, considering the noise power level available in the spectrum analyzer data. The user also determines the interval in the frequency domain over which the integration will be performed.

3. The area in the frequency domain below the interferer signal and above the reference power level used in the previous step is calculated. The same frequency interval as in the previous step is used for the integration.

4. The I-factor is obtained by dividing the area below the intersection of the two signals by the area below the interferer signal.

In the second step of the above procedure, we assume that the receiver radio uses a filter similar to its TSM and we estimate this filter using its transmit power distribution over the frequency domain. With this estimation, we calculate the interferer power falling below the measured (estimated) filter, as in Figure 1a.

If we have a better estimation for the filter being used by the receiver radio, there is no need to collect the traces for the receiver in the first step. Instead, after collecting the interferer traces, we can directly calculate the ratio of the interferer signal power falling below this filter to the total interferer power to estimate the I-factor, as in Figure 1b.

Algorithm 1 outlines the steps of our proposed method. The output of the algorithm, $I\left(c h_{\text {int }}, c h_{\text {recv }}\right)$, is the Ifactor calculated between channels $c h_{\text {intf }}$ and $c h_{\text {recv }}$. If there is an analytical model for the receiver bandpass filter's frequency response, such as the one in (5), it must be converted into a suitable representation for processing. In our implementation, this corresponds to adding the filter model onto the bitmap where the interferer signal trace resides, using a different color than the interferer signal trace's color. To calculate the total interferer power and the amount of interferer power that overlaps with the receiver radio's filter on the frequency interval $\left[f_{l}, f_{u}\right]$, we use the procedure given in Algorithm 2. The inputs $\left[f_{l}, f_{u}\right]$ and referencePowerLevel should be specified in terms of the pixels of the bitmap $B$.

Algorithm 1 SIAM I-factor model and computation method Input: $F_{c, i}$. The center frequency of the interferer channel, $i$. Input: $F_{c, j}$. The center frequency of the receiver channel, $j$. Output: $I(i, j)$

1: Collect spectrum analyzer traces for the interferer signal on channel $i$

2: if No model is assumed for the receiver filter's frequency response then

3: $\quad$ Collect spectrum analyzer traces on the receiver channel $j$

4: else

5: $\quad$ Convert the receiver filter model into suitable representation

6: end if

7: $\left[f_{l}, f_{u}\right] \Leftarrow$ User input $\quad \triangleright f_{l}$ and $f_{u}$ are the limits of integration

8: totalinterfererPower $\Leftarrow \int_{f_{l}}^{f_{u}} P_{\text {intf }}\left(f, F_{c, i}\right) d f$

$\triangleright$ Approximated with Algorithm 2

9: overlapPower $\Leftarrow \int_{f_{l}}^{f_{u}} \min \left\{P_{\text {intf }}\left(f, F_{c, i}\right), P_{\text {recv_ch }}\left(f, F_{c, j}\right)\right\} d f$ $\triangleright$ Approximated with Algorithm 2

10: $I(i, j) \Leftarrow \frac{\text { overlapPower }}{\text { totallnterfererPower }}$

\section{Algorithm 2 Total interferer power and overlap power} calculation on bitmap

Input: Bitmap $B$ containing the trace of the receiver channel signal (or the receiver filter's frequency response curve) together with the trace of the interferer signal. The traces should be in different colors (i.e., Color recv and Color $_{\text {intf }}$ ).

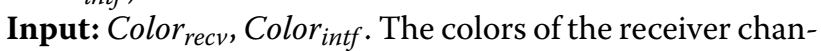
nel signal trace (or the frequency response curve) and the interferer signal trace, respectively.

Input: referencePowerLevel. Power above referencePowerLevel is summed.

Input: $\left[f_{l}, f_{u}\right]$. The frequency bounds of the interval of integration.

Output: totalInterfererPower, overlapPower

1: totalinterfererPower $\Leftarrow 0$

2: overlapPower $\Leftarrow 0$

3: Draw line $y=$ referencePowerLevel on bitmap $B$ with color Color $r_{\text {ref }}$, such that Color $_{\text {ref }} \notin\left\{\right.$ Color $_{\text {recv }}$, Color $\left._{\text {intf }}\right\}$

4: for $f=f_{l}$ to $f_{u}$ do

5: $\quad$ Power $_{\text {recv }} \Leftarrow 0$

6: $\quad$ Power $_{\text {intf }} \Leftarrow 0$

7: $\quad$ for $p=1$ to height $[B]$ do $\quad \triangleright \operatorname{height~}[B]$ is the

8: $\quad$ if $\operatorname{color}\left[P_{f, p}\right]=$ Color $_{\text {rec }}$ then height of the bitmap $\triangleright \operatorname{color}\left[P_{f, p}\right]$ is the color of pixel $P_{f, p}$

9: $\quad$ Power $_{\text {recv }} \Leftarrow($ referencePowerLevel $-p)$ 

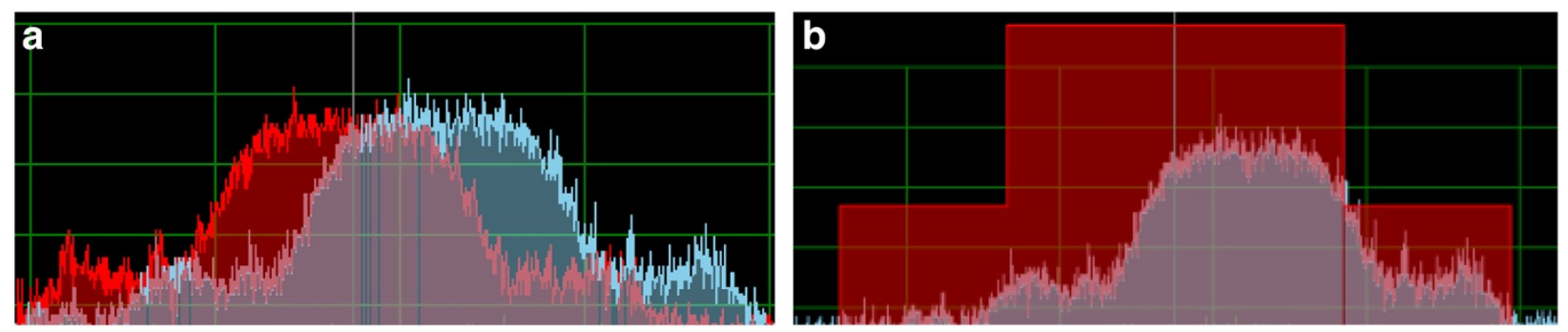

Figure $1 \mathrm{I}$-factor can be modeled when no analytical model is given for the receiver filter's frequency response by estimating the filter by the TSM as in Figure 1a. When an analytical model is assumed for the receiver filter's frequency response, the l-factor can be modeled without the need for receiver channel traces, as in Figure 1b. (a) Two DSSS signals shown together. The receiver filter may be estimated using a transmission on the receiver's channel (channel 6 here, shown in red). The blue trace belongs to an interferer on the adjacent channel 7. (b) The receiver filter, in red, estimated as the DSSS TSM centered on channel 6 and the captured interferer DSSS signal, in blue, on channel 7, shown together.

else if $\operatorname{color}\left[P_{f, p}\right]=$ Color $_{\text {intf }}$ then

Power $_{\text {intf }} \Leftarrow($ referencePowerLevel $-p)$ else if $\operatorname{color}\left[P_{f, p}\right]=$ Color $_{\text {ref }}$ then

\section{break}

end if

end for

if Power $_{\text {intf }} \neq 0$ then

totalInterfererPower $\Leftarrow$ totalInterfererPower +

Power $_{\text {intf }}$

if Power $_{\text {rec }} \neq 0$ then $\quad \triangleright$ Overlap region

overlapPower $\Leftarrow$ overlapPower + $\min \left\{\right.$ Power $_{\text {recv }}$, Power $\left._{\text {intf }}\right\}$

end if

end if

nd for
The method we propose above defines I-factor as the ratio of the area below the intersection of two frequency domain signal traces to the total area below the trace that belongs to the interferer signal. We now discuss another definition for the I-factor that has a more concrete physical interpretation. Equation (4), introduced earlier, models the percentage of the maximum interference energy radiated on channel $i$ and received by a receiver on channel $j$, where $F_{c, i}$ is the center frequency of channel $i$ and $F_{c, j}$ is the center frequency of channel $j$. The maximum interference energy is defined as the interference energy that would be received by another receiver operating on channel $i$ in the same location as the receiver on channel $j$.

Algorithm 3 outlines the steps of I-factor calculation based on (4). We call this method described with

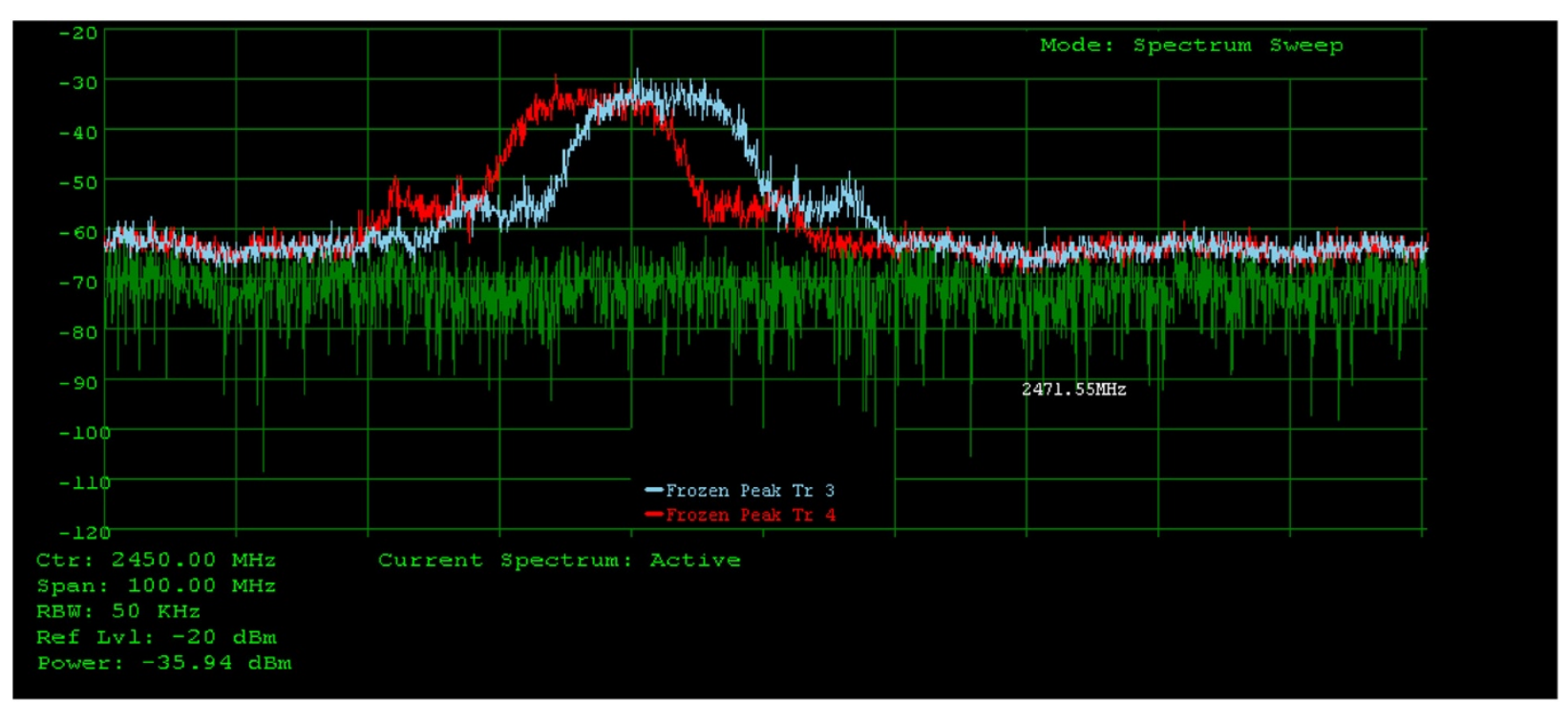

Figure 2 Signal traces showing overlap between transmitted signals on channels 6 (red trace) and 7 (blue trace). 


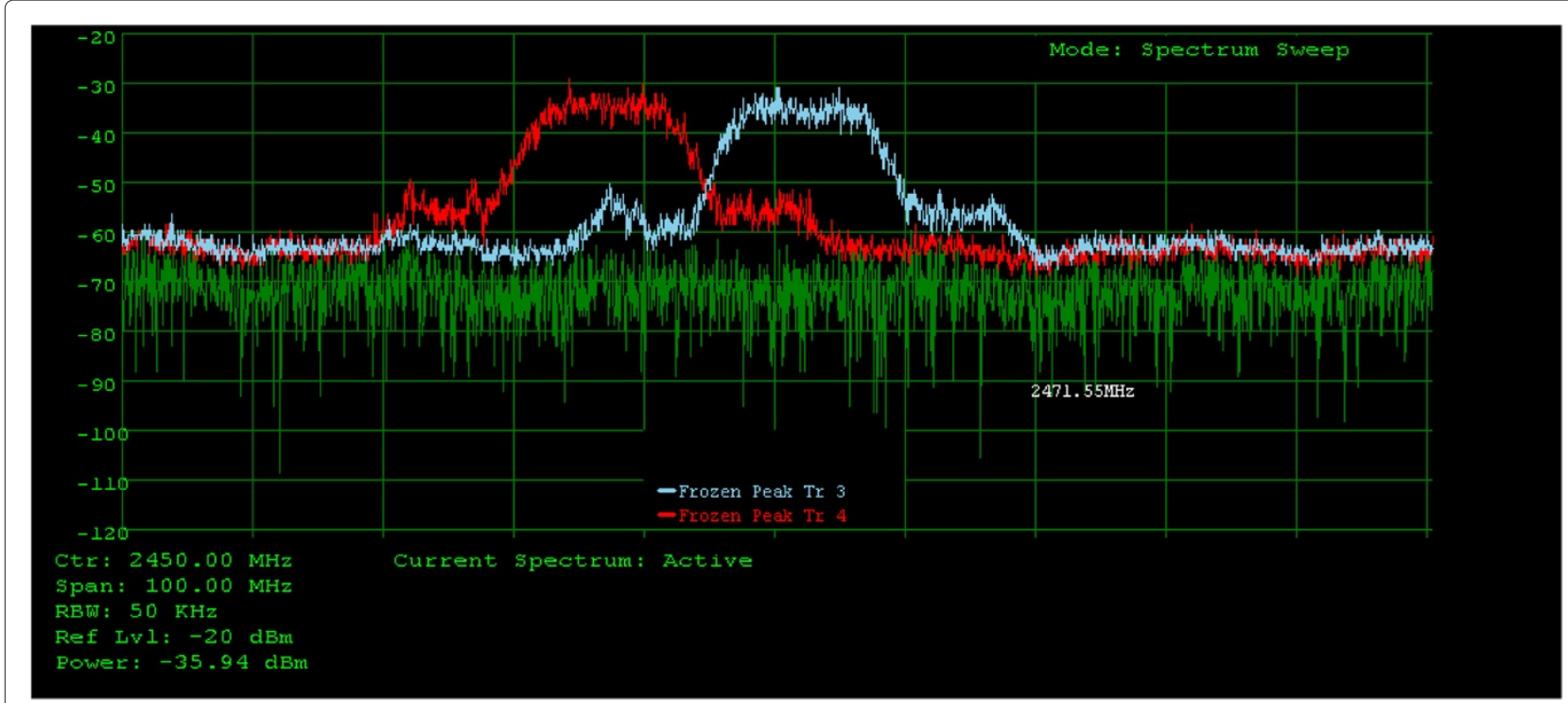

Figure 3 Signal traces showing overlap between transmitted signals on channels 6 (red trace) and 9 (blue trace).

Algorithm 3 as the PMIE method: Percentage of Maximum Interference Energy. While calculating totalReceivedIntfEn and maximumIntfEn, Algorithm 4 is called twice, first with a receiver channel signal trace (or the receiver filter's frequency response centered around $\left.F_{c, j}\right)$ and then with an interferer channel signal trace (or the receiver filter's frequency response centered around $\left.F_{c, i}\right)$.

Algorithm 3 PMIE I-factor model and computation method Input: $F_{c, i}$. The center frequency of the interferer channel, $i$.
Input: $F_{c, j}$. The center frequency of the receiver channel, $j$. Output: $I(i, j)$

1: Collect spectrum analyzer traces for the interferer signal on channel $i$

2: if No model is assumed for the receiver filter's frequency response then

3: $\quad$ Collect spectrum analyzer traces on the receiver channel $j$

4: $\quad\left[f_{l}, f_{u}\right] \Leftarrow$ User input $\quad \triangleright f_{l}$ and $f_{u}$ are the limits of

5: else

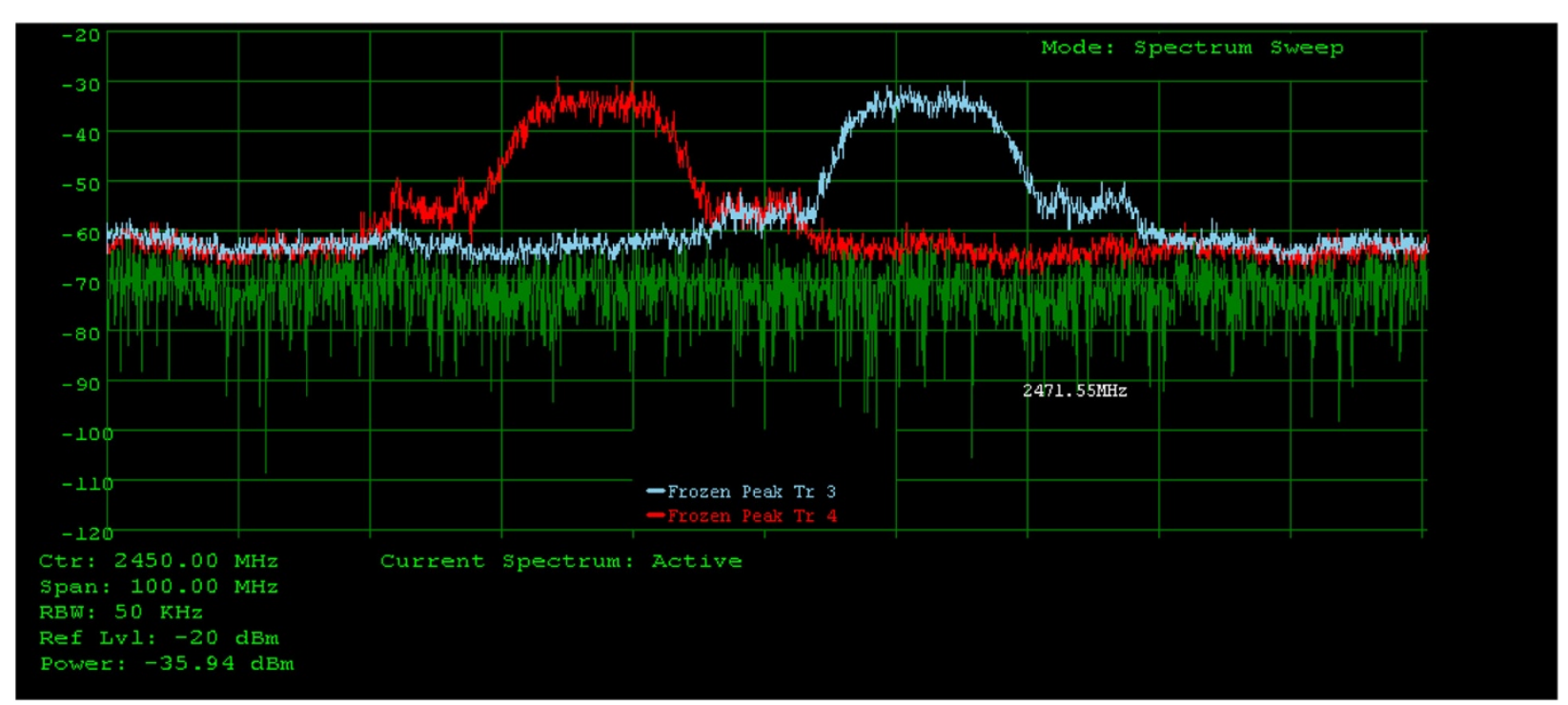

Figure 4 Signal traces showing overlap between transmitted signals on channels 6 (red trace) and 11 (blue trace). 


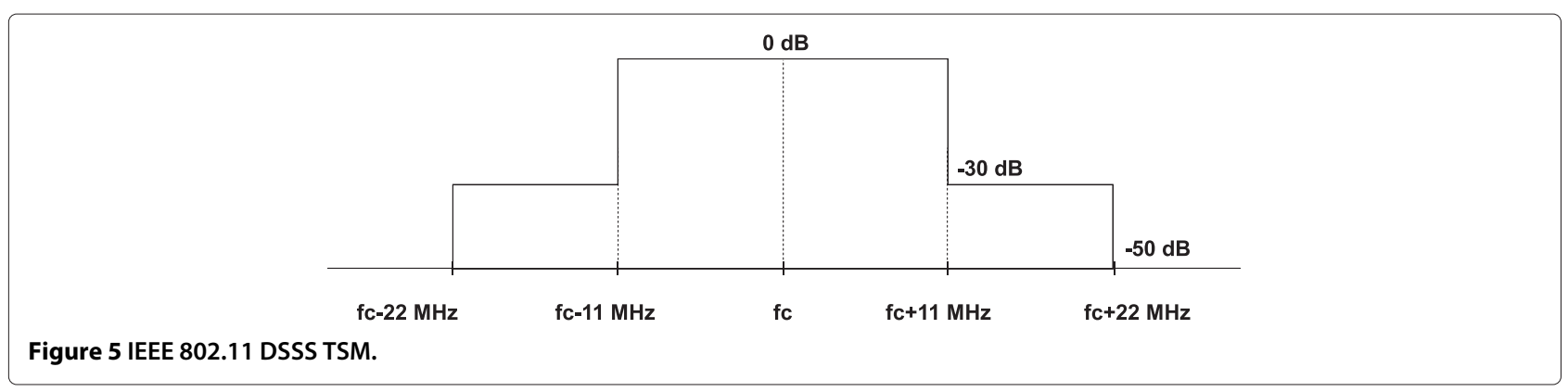

6: $\quad$ Convert the receiver filter model into suitable representation

7: $\quad f_{l} \Leftarrow F_{c, j}-\frac{B w}{2} \quad \triangleright B w$ is the width of the bandpass filter's frequency response

8: $\quad f_{u} \Leftarrow F_{c, j}+\frac{B w}{2}$ $\triangleright f_{l}$ and $f_{u}$ are the limits of integration

9: end if

10: totalReceivedIntfEn $\Leftarrow \int_{f_{l}}^{f_{u}} P_{\text {intf }}\left(f, F_{c, i}\right) B_{R}\left(f, F_{c, j}\right) d f$ $\triangleright$ Approximated with Algorithm 4 11: maximumIntfEn $\Leftarrow \int_{f_{l}+\left(F_{c, i}-F_{c, j}\right)}^{f_{u}+\left(F_{c, i}-F_{c, j}\right)} P_{\text {intf }}\left(f, F_{c, i}\right) B_{R}\left(f, F_{c, i}\right) d f$ 12: $I(i, j) \Leftarrow \frac{\text { totalReceivedIntfEn }}{\text { maximumIntfEn }}$

$\triangleright$ Approximated with Algorithm 4

\section{Algorithm 4 Received energy calculation on bitmap}

Input: Bitmap $B$ containing the trace of the receiver channel signal (or the receiver filter's frequency response curve) together with the trace of the interferer signal. The traces should be in different colors (i.e., Color recv and Color $\left._{\text {intf }}\right)$.
Input: Color $_{\text {recv }}$, Color intf. The colors of the receiver channel signal trace (or the frequency response curve) and the interferer signal trace, respectively.

Input: $\left[f_{l}, f_{u}\right]$. The frequency bounds of the interval of integration.

\section{Output: totalReceivedEn}

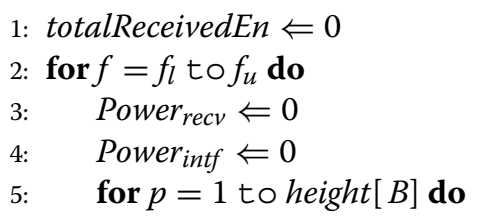

6:

7: 8: 9: 10 : 11: $\triangleright$ height $[B]$ is the height of the bitmap then $\triangleright \operatorname{color}\left[P_{f, p}\right]$ is the color of pixel $P_{f, p}$ Power $_{\text {recv }} \Leftarrow 10 \log (p / 10)$ else if $\operatorname{color}\left[P_{f, p}\right]=$ Color $_{\text {intf }}$ then end if Power $_{\text {intf }} \Leftarrow 10 \log (p / 10)$ end for

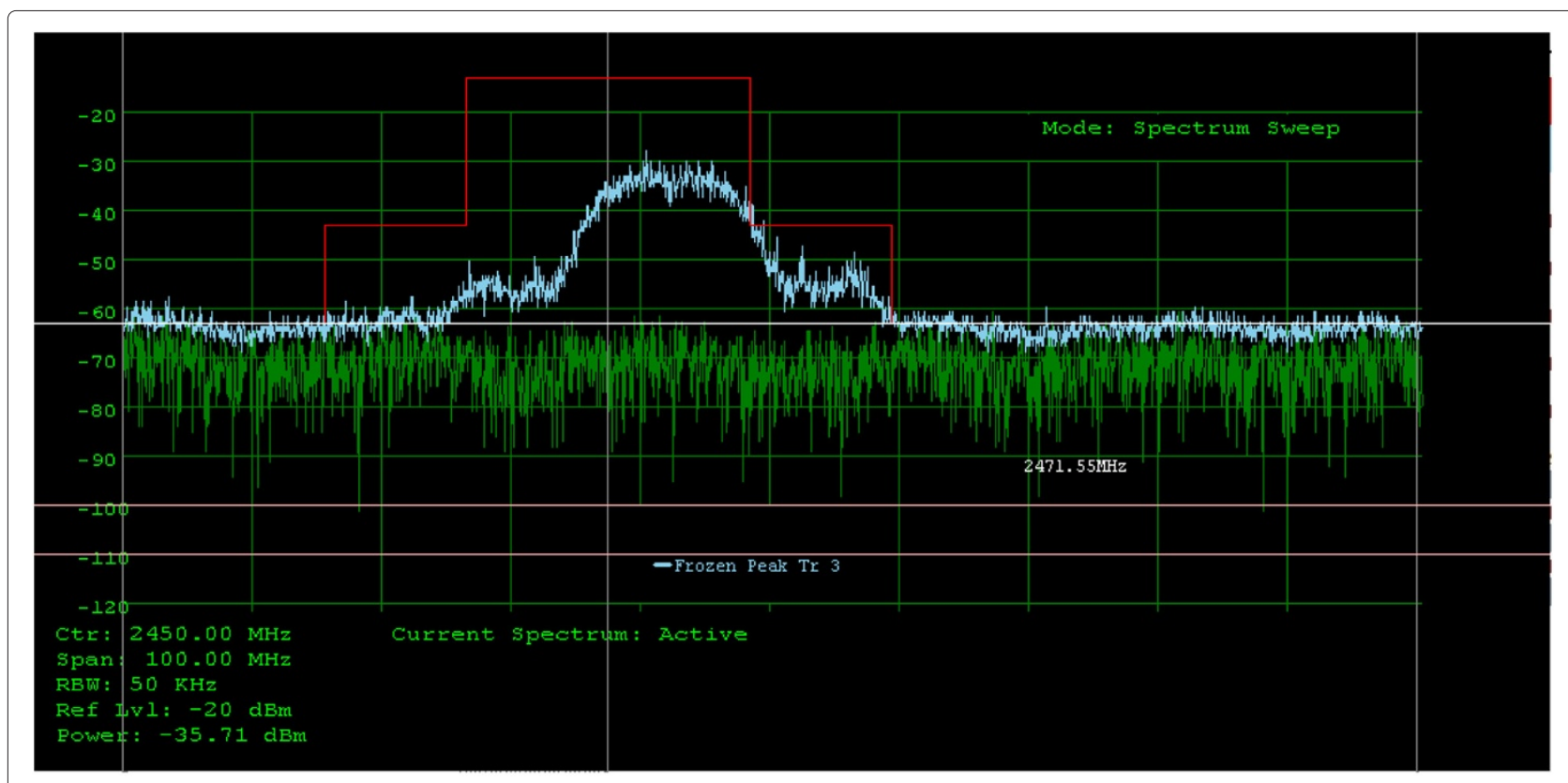

Figure 6 TSM on channel 6 (red) and signal trace on channel 7 (blue). 


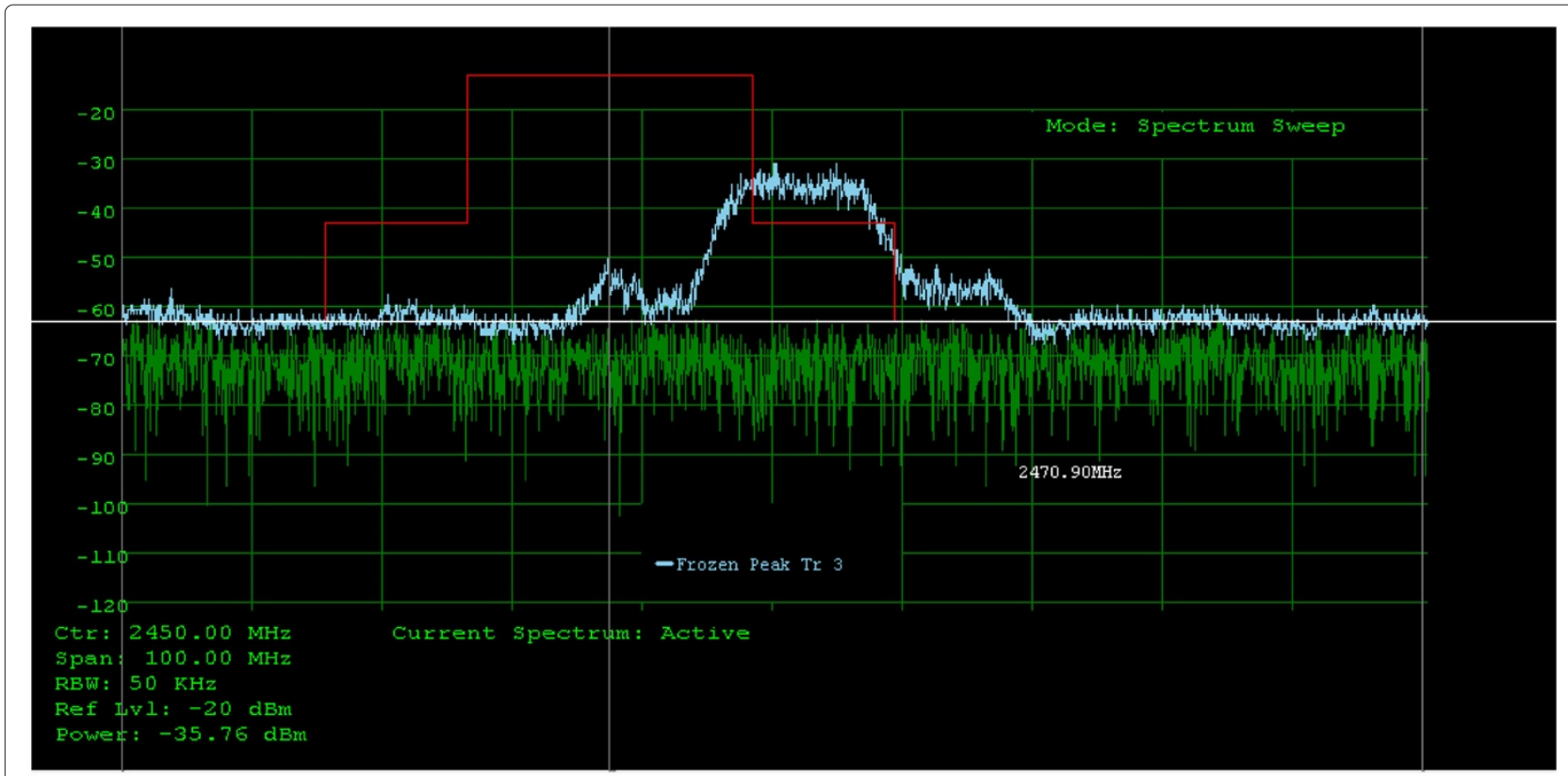

Figure 7 TSM on channel 6 (red) and signal trace on channel 9 (blue).

\section{2: $\quad$ totalReceivedEn $\Leftarrow$ Power $_{\text {recv }}$ Power $_{\text {intf }}$ 13: end for}

We implement these novel methods for modeling and computing the I-factor using the Java programming language. The current implementation requires the signal traces on the receiver's channel and on the interferer's channels to be placed on the same bitmap using different colors, as in Figures 2, 3, and 4. Using the graphical user interface (GUI) of the Java program, the user is able to determine the reference power level and the integration interval in the frequency domain. The program outputs the ratio of the intersection area to the total power of the first signal and the ratio of the intersection area to the total power of the second signal. I-factor values with respect to a receiver operating on the first signal's channel are the ratios of the intersection areas to the second signal's total

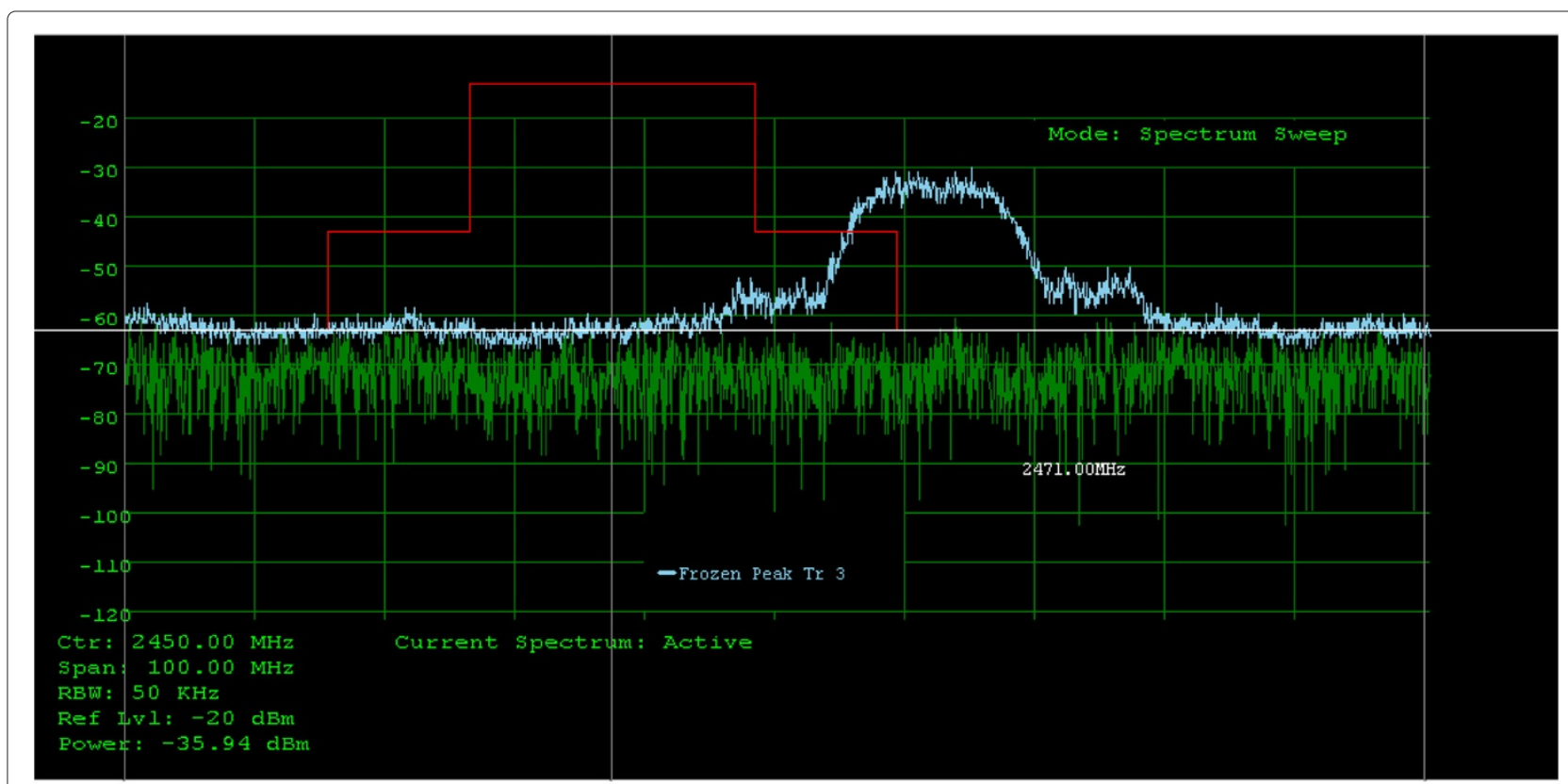

Figure 8 TSM on channel 6 (red) and signal trace on channel 11 (blue). 
Table 1 Interference factors calculated using SIAM and PMIE (see Figures 2, 3, 4, 6, 7, 8 and 10, 11, 12, 13) and compared with some of the existing models in the literature

\begin{tabular}{|c|c|c|c|c|c|}
\hline $\begin{array}{c}\text { Ch. } \\
\text { separation }\end{array}$ & $\begin{array}{l}\text { I-factor based on } \\
\text { signal traces } \\
\text { (SIAM) }\end{array}$ & $\begin{array}{l}\text { I-factor based on } \\
\text { TSM assumption } \\
\text { (SIAM) }\end{array}$ & $\begin{array}{l}\text { I-factor based on } \\
\text { signal traces } \\
\text { (PMIE) }\end{array}$ & $\begin{array}{l}\text { I-Factor based on } \\
\text { analytical model }\end{array}$ & $\begin{array}{l}\text { I-factor based on } \\
\text { SNR Measurements }\end{array}$ \\
\hline 0 & - & - & 1 & - & - \\
\hline 1 & 0.61 & 0.82 & 0.68 & 0.60 & 0.96 \\
\hline 2 & 0.33 & 0.56 & 0.18 & 0.30 & 0.77 \\
\hline 3 & 0.14 & 0.27 & 0.008 & 0.11 & 0.66 \\
\hline 4 & 0.09 & 0.1 & 0.003 & 0.01 & 0.39 \\
\hline 5 & 0.03 & 0.03 & 0.002 & 0 & 0 \\
\hline 6 & 0.01 & 0.01 & 0.002 & - & - \\
\hline
\end{tabular}

power. Likewise, I-factor values with respect to a receiver operating on the second signal's channel are the ratios of the intersection areas to the first signal's total power.

PMIE has a more concrete physical interpretation when compared to SIAM because PMIE defines the I-factor as the ratio of the total received interference energy from an interferer on the receiver's channel to the total received energy from the same interferer on the interferer's channel (the maximum interference energy). PMIE I-factor value is, consequently, strictly 1 when the receiver captures all of the interferer's energy and 0 when the receiver filters all of the interferer's energy. However, because of the way SIAM is defined, these may not always hold for SIAM I-factor values. PMIE, on the other hand, requires the knowledge of the receiver radio's filter (the $B_{R}$ function). If $B_{R}$ is not known, SIAM, however, can approximate it from transmissions on the receiver radio's channel by a transmitter radio using the same wireless standard and PHY layer specification as the receiver radio. This gives more flexibility to SIAM and makes it possible to obtain approximated I-factor values using SIAM when $B_{R}$ is not known. If $B_{R}$ is known, PMIE can be used to obtain more accurate I-factor values.

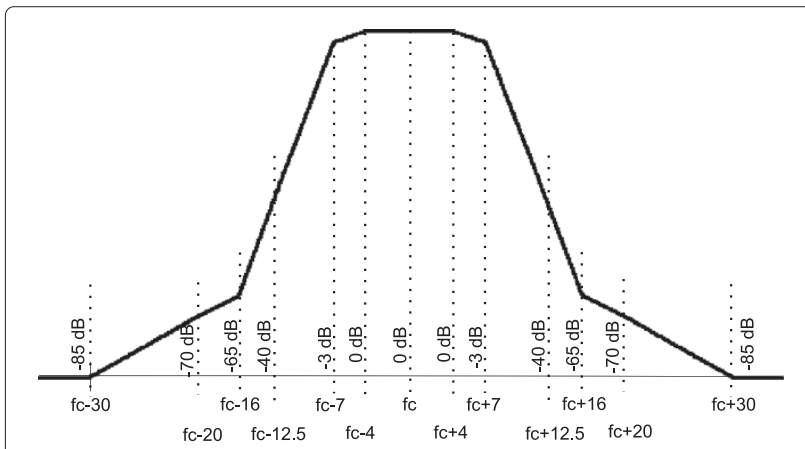

Figure 9 Receiver filter's frequency response of the Maxim MAX2820/MAX2821 802.11b transceiver. $f_{c}$ denotes the center frequency, and the unit of the $x$-axis is $\mathrm{MHz}$
Our measurement results for the interference factor between two DSSS signals and between the DSSS TSM and an interferer DSSS signal are given in Section 5.1. We have written a separate Java program to implement PMIE (Algorithms 3 and 4). In Section 5.2, we report on the Ifactor calculations using the PMIE method. In Section 5.3, we report our results on the I-factor values between an 802.15.4 OQPSK interferer and an 802.11 DSSS receiver.

\section{Measurement results and comparisons}

\subsection{Measurements for modeling interference between} 802.11 DSSS signals using SIAM

We collect 802.11 b DSSS signal traces (see Figures 2, 3, and 4) with the $2.4 \mathrm{GHz}$ spectrum analyzer. For collecting these traces, one radio is kept fixed at channel 6 $(2437 \mathrm{MHz})$ and the other radio is swept from channel 7 $(2442 \mathrm{MHz})$ to channel $12(2467 \mathrm{MHz})$ of the 14 channels defined by the standard. In this case, we assume that the receiver filter for the specific DSSS radio in use is neither known, nor can it be estimated analytically. We also consider the case where we approximate the receiver filter using the DSSS TSM, depicted in Figure 5 and defined as follows:

$$
\operatorname{TSM}\left(f, F_{c}\right)= \begin{cases}-50 \mathrm{dBr} & \text { if }\left|f-F_{c}\right|>22 \mathrm{MHz} \\ -30 \mathrm{dBr} & \text { if } 11<\left|f-F_{c}\right|<22 \mathrm{MHz} \\ 0 \mathrm{dBr} & \text { Otherwise. }\end{cases}
$$

Here, $f$ denotes the frequency and $F_{c}$ is the center frequency of the receiver channel. With this approximation, there is no need to collect traces for a transmitted signal on the receiver's channel; it suffices to collect traces for the interferer radio. Figures 6, 7, and 8 show selected data for these measurements. In these measurements, the receiver is kept fixed at channel 6 (center frequency at $2437 \mathrm{MHz}$ ) and the interferer sweeps from channel 7 to channel 12 . Only the interferer signal traces need be collected; the 


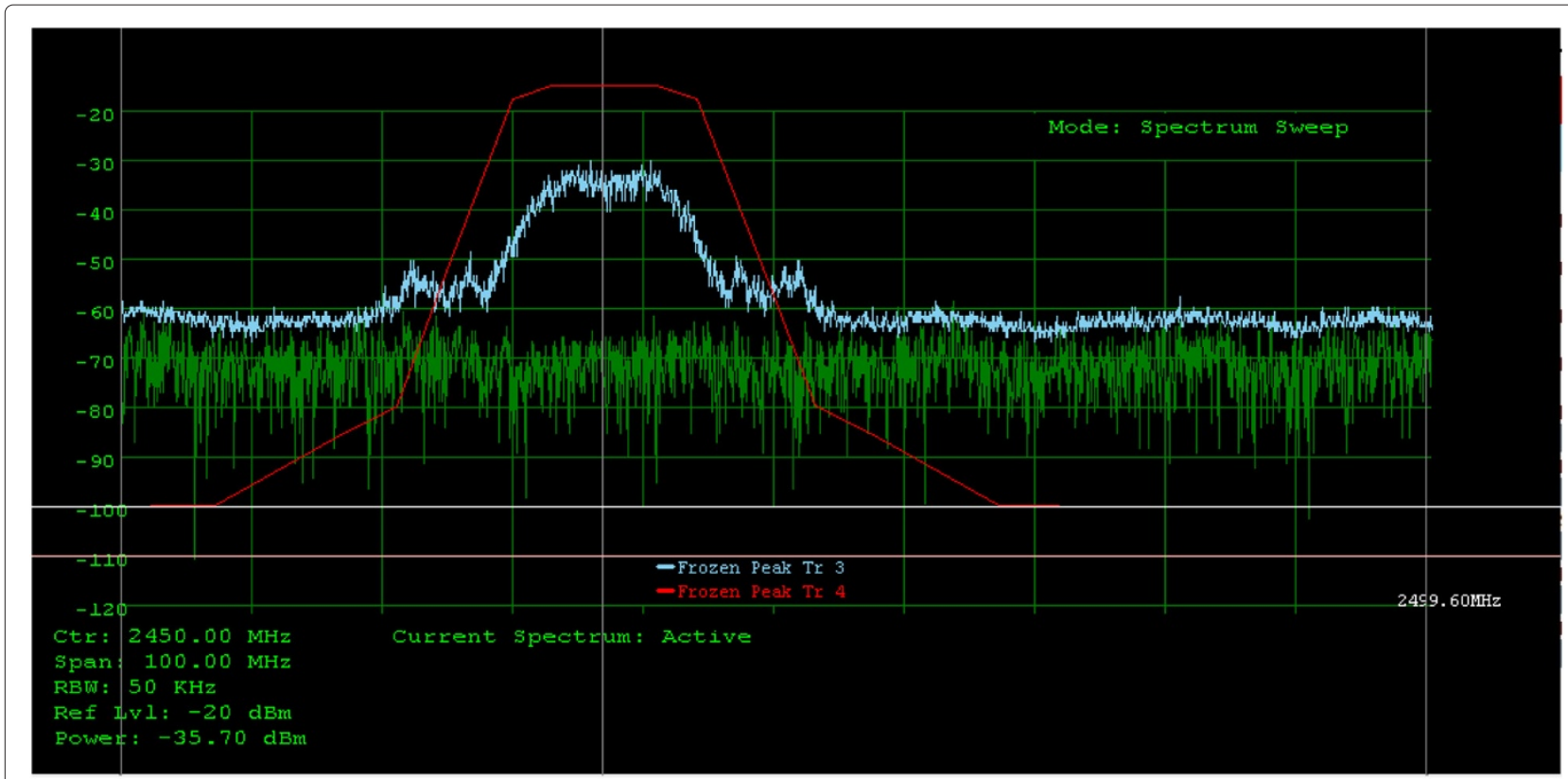

Figure 10 MAX2820 receiver filter's frequency response on channel 6 (red) and signal trace on channel 6 (blue).

receiver filter's frequency response curve is approximated by the 802.11 DSSS TSM centered at channel 6 , shown in red color in these figures.

The I-factor values calculated for the above two cases using SIAM are given in the second and third columns of Table 1. In the fifth column, we give the estimations of the I-factor for DSSS radios using the analytical model proposed in [1]. In this model, the receiver filter's frequency response $\left(B_{R}\left(f, F_{c, r}\right)\right.$ in (2)) is assumed to be identical to the DSSS TSM and is given in $(5)\left(B_{R}\left(f, F_{c, r}\right)=\right.$ $\left.\operatorname{TSM}\left(f, F_{c, r}\right)\right)$. The last column of Table 1 gives the SNR measurement-based estimation of the I-factor values as reported in [3], measured for the cases where the receiver is fixed on channel 6 and the transmitter is operated on

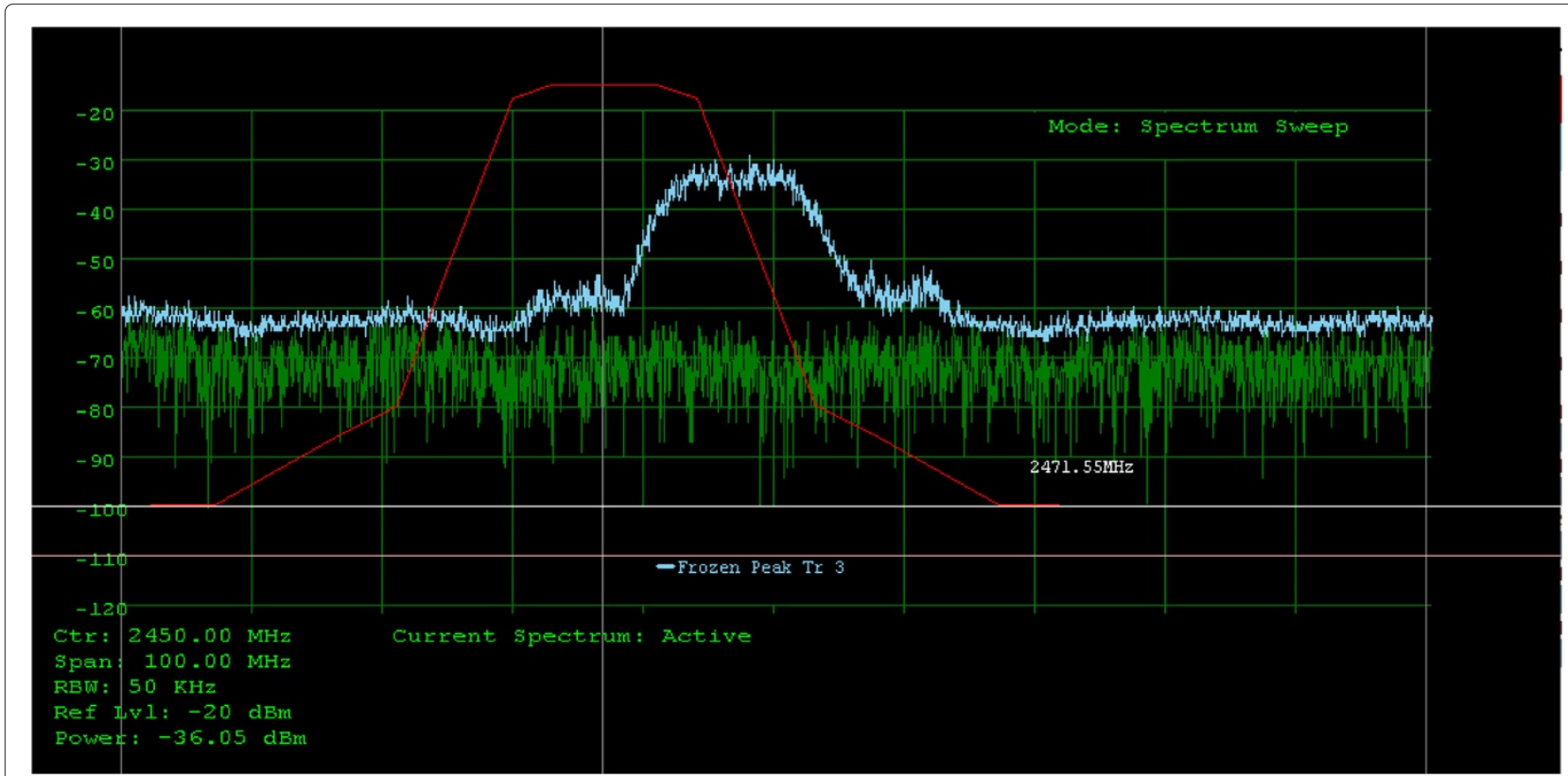

Figure 11 MAX2820 receiver filter's frequency response on channel 6 (red) and signal trace on channel 8 (blue). 


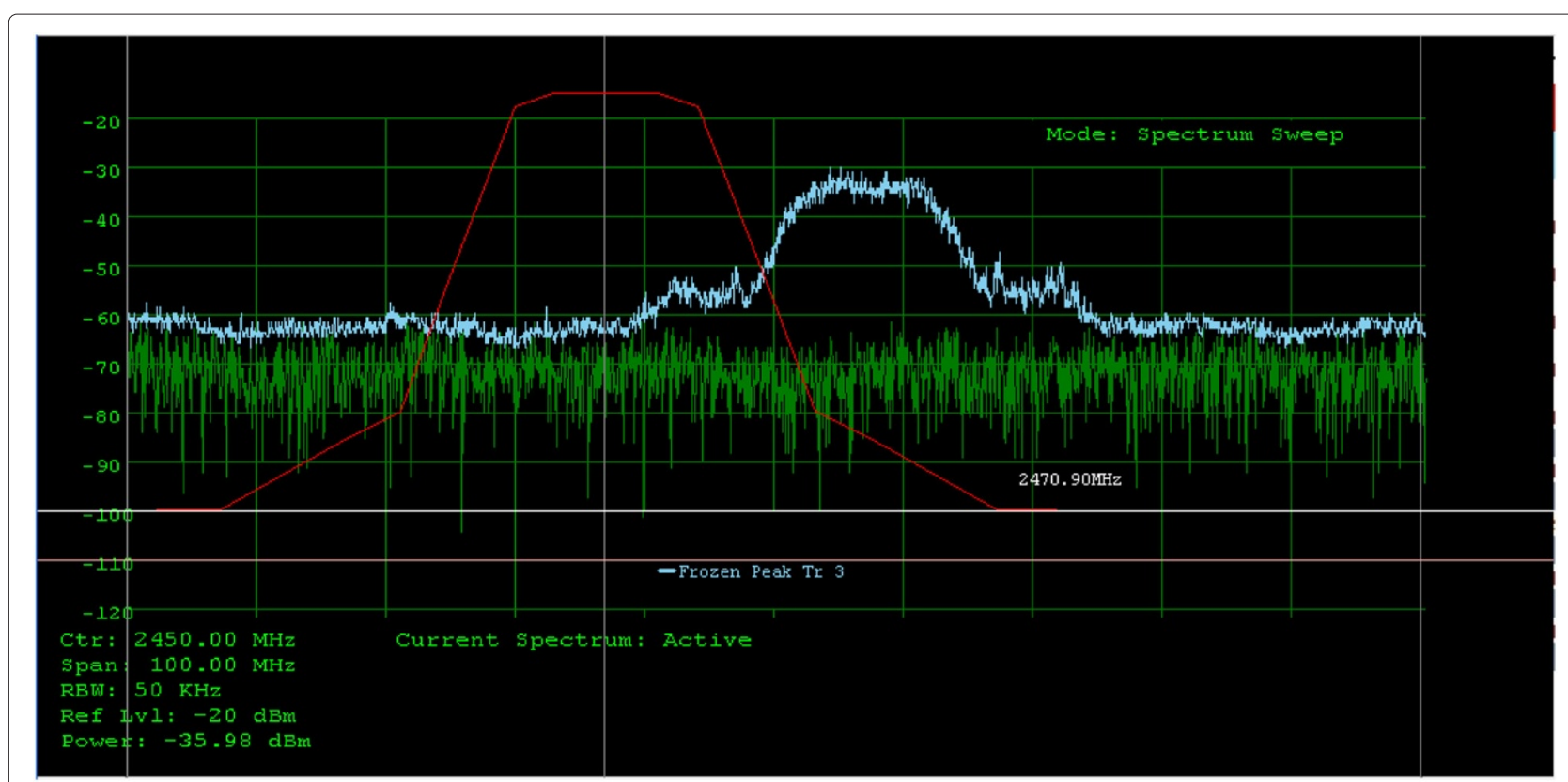

Figure 12 MAX2820 receiver filter's frequency response on channel 6 (red) and signal trace on channel 10 (blue).

channels 7 to 11 . These transmitter channels match the channels used in our experiments.

\subsection{Measurements for modeling interference between 802.11 DSSS signals using the PMIE method}

Using the collected interferer 802.11 DSSS signal traces and the receiver filter's frequency response of the Maxim MAX2820/2821 802.11b transceiver [13] given in Figure 9, we calculate the I-factors between 802.11 DSSS channels using Algorithms 3 and 4 (PMIE). Figures 10,11, 12, and 13 show selected data used for these calculations. For the related measurements, the receiver is kept fixed at channel 6 (represented by the frequency response curve in red centered at $2437 \mathrm{MHz}$ ) and the interferer sweeps from channel 6 to channel 12 . For this setup, only the interferer signal traces need be collected; the receiver filter's frequency response curve is generated by our software. The fourth column of Table 1 summarizes the I-factor

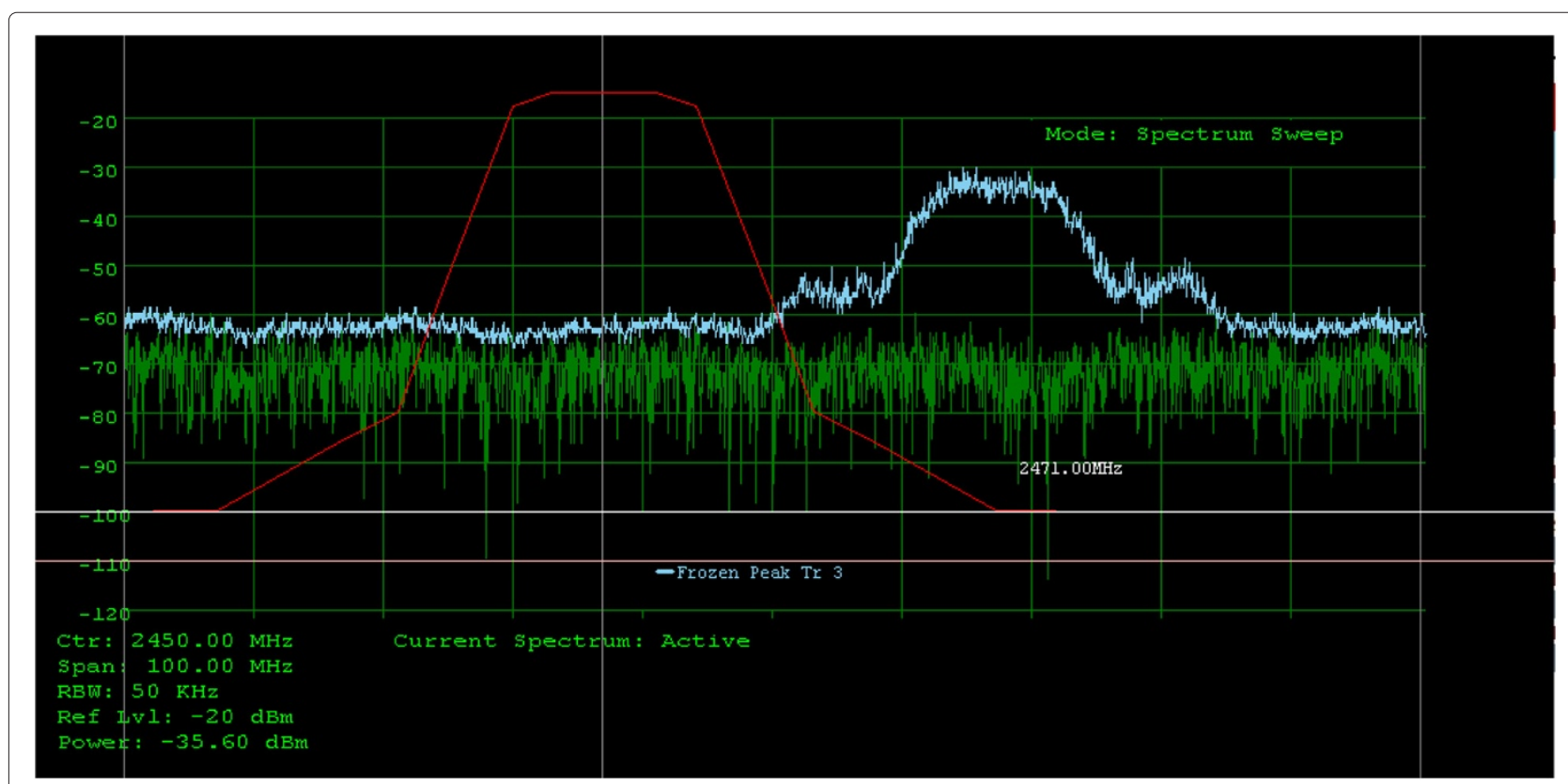

Figure 13 MAX2820 receiver filter's frequency response on channel 6 (red) and signal trace on channel 12 (blue). 


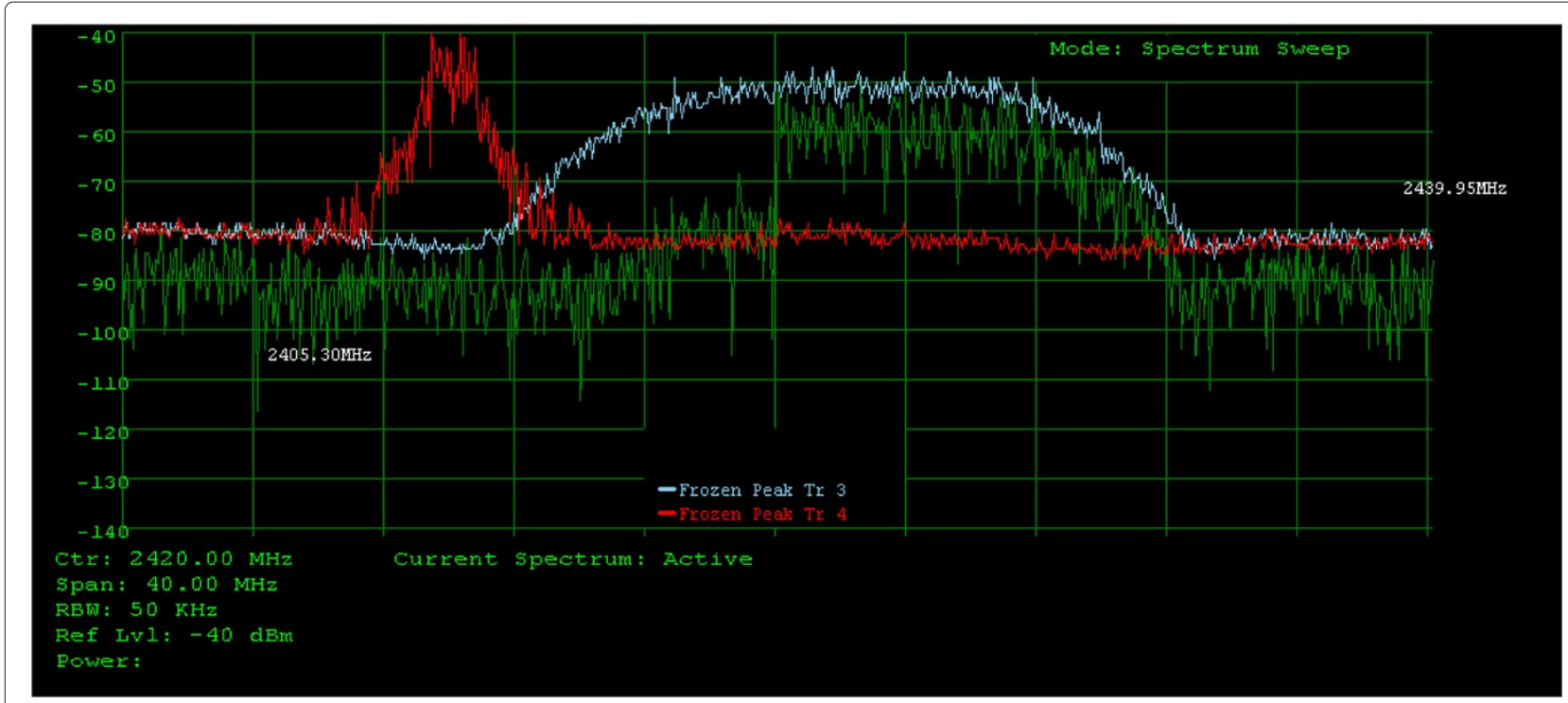

Figure 14 Signal traces showing overlap between 802.11 channel 3 (blue) and 802.15.4 channel 12 (red).

calculations for this setup. Compared to the third column, which uses the 802.11 DSSS TSM and SIAM, it can be observed that the I-factor values obtained using the narrower MAX2820/2821 filter frequency response and the PMIE method drop faster as channel separation increases. Further, by the definition of I-factor in (4), we have an Ifactor exactly equal to 1.0 when the channel separation is 0 .

\subsection{Measurements for modeling interference between 802.11 DSSS and 802.15.4 OQPSK signals}

To model the interference between an 802.15.4 (ZigBee) transmitter and an $802.11 \mathrm{~b}$ receiver, we perform another set of experiments and take measurements with our spectrum analyzer. In this set, we use five Crossbow TelosB motes [14] and two 802.11b radios in ad-hoc (IBSS) mode. The TelosB motes have IEEE 802.15.4 compliant Texas Instruments CC2420 radios with integrated onboard antennas, and communicate with a data rate of $250 \mathrm{Kbps}$. They are commonly employed in wireless sensor networks. One of the TelosB motes is used as the base station in our experiment. The other four motes periodically sample their sensors and transmit their readings to the base station. We use four ZigBee transmitters to increase the chance of an 802.15.4 signal being registered on the spectrum analyzer. The $802.11 \mathrm{~b}$

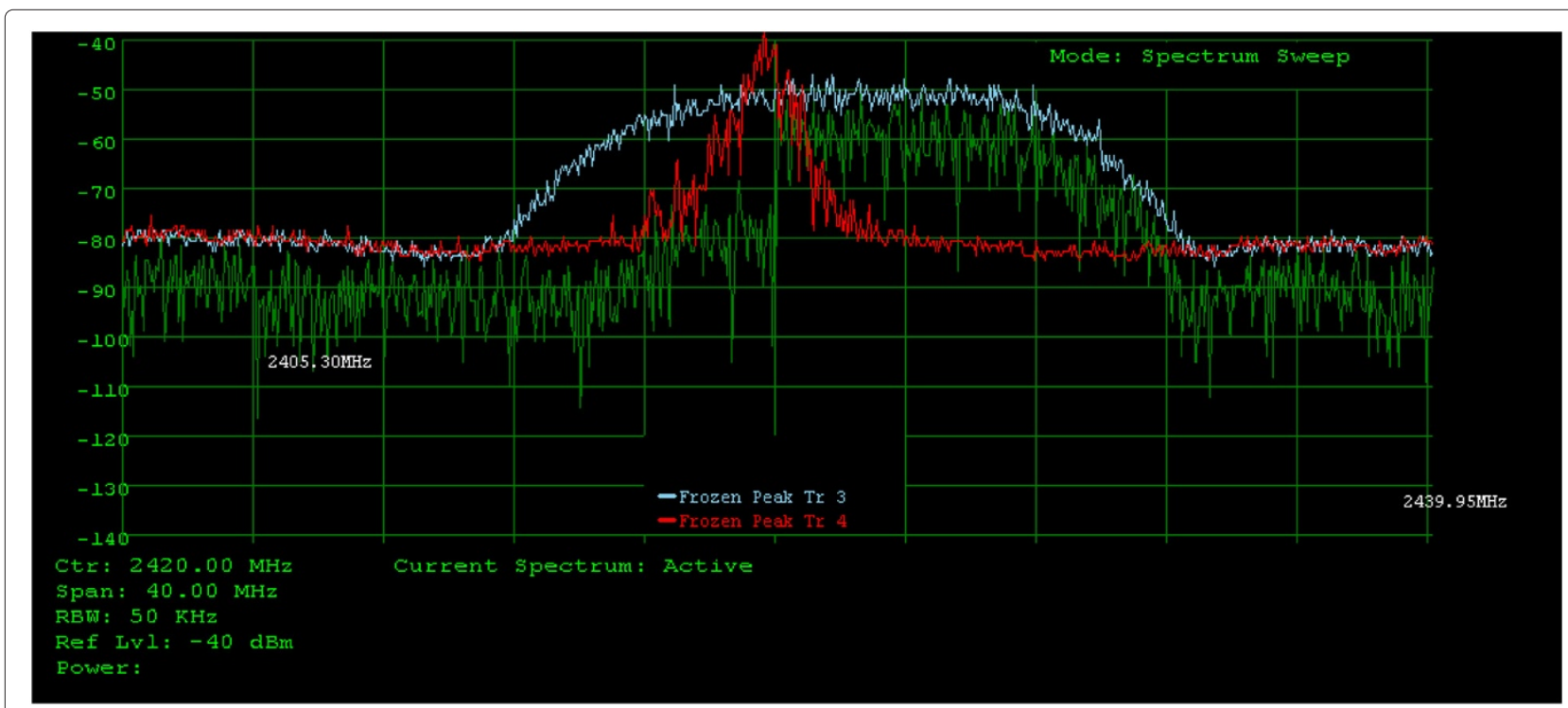

Figure 15 Signal traces showing overlap between 802.11 channel 3 (blue) and 802.15 .4 channel 14 (red). 


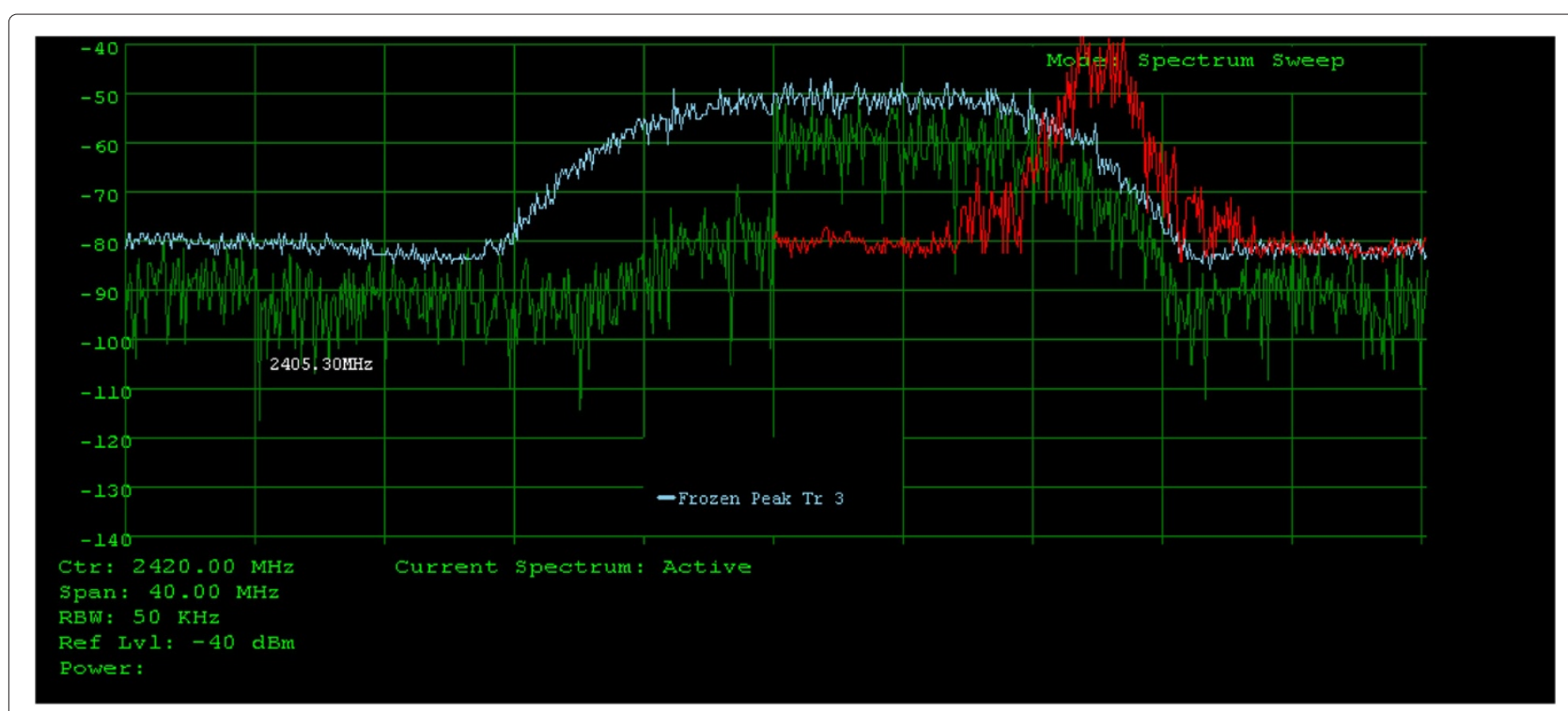

Figure 16 Signal traces showing overlap between 802.11 channel 3 (blue) and 802.15.4 channel 16 (red).

radios are operated on (802.11) channel 3, whose center frequency is $2422 \mathrm{MHz}$. The channel of the ZigBee network is varied from (802.15.4) channel 11 (central frequency $2405 \mathrm{MHz}$ ) to channel 17 (central frequency $2435 \mathrm{MHz})$.

The IEEE 802.15.4 standard [15] defines channels in the $868 / 915 \mathrm{MHz}$ and $2450 \mathrm{MHz}$ spectra. The frequency band in the $868 \mathrm{MHz}$ spectrum is narrow, starting at $868 \mathrm{MHz}$ and ending at $868.6 \mathrm{MHz}$; and the frequency band in the $915 \mathrm{MHz}$ spectrum starts at 902 and ends at $928 \mathrm{MHz}$. The frequency band that we observe in our experiments is in the $2.4 \mathrm{GHz}$ spectrum, and starts at $2400 \mathrm{MHz}$, and ends at 2483.5 MHz. The IEEE 802.15.4 2006 standard specifies channels with a combination of a channel page and a channel number. For channel page 0 , the standard defines 16 channels in the $2450 \mathrm{MHz}$ band, 10 channels in the $915 \mathrm{MHz}$ band, and one channel in the $868 \mathrm{MHz}$ band. Each consecutive 802.15.4 channel on channel page 0 in the $2450 \mathrm{MHz}$ band is separated by $5 \mathrm{MHz}$, and the center frequency, $F_{c}$, for channel $k$ is given by [15]:

$$
F_{c}=2405+5(k-11), k=11,12, \ldots, 26,
$$

where $F_{c}$ is in $\mathrm{MHz}$. The first channel on channel page 0 in the $2450 \mathrm{MHz}$ band is channel 11 and the last channel is channel 26.

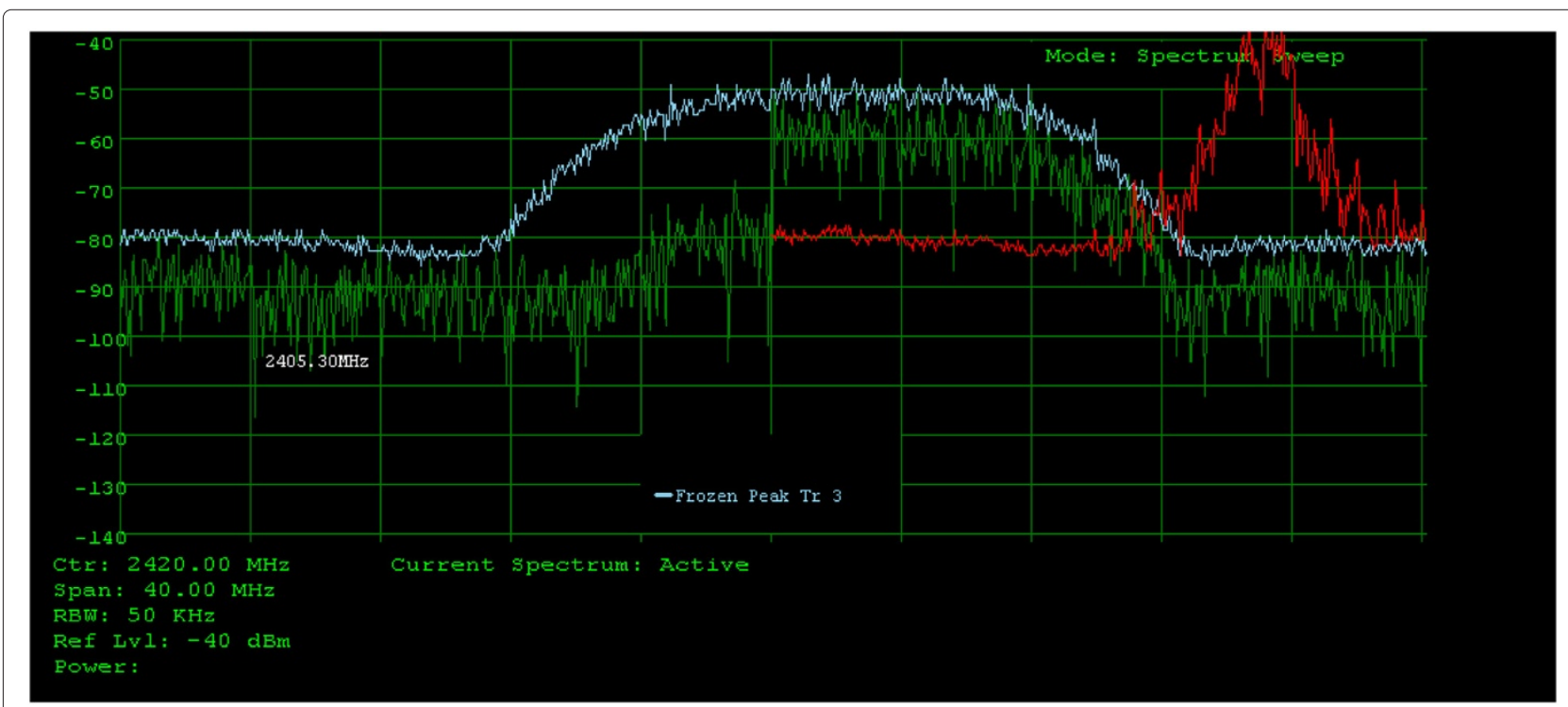

Figure 17 Signal traces showing overlap between 802.11 channel 3 (blue) and 802.15 .4 channel 17 (red). 
Table 2 Interference factors calculated using SIAM (see Figures 14, 15, 16, and 17)

\begin{tabular}{ccc}
\hline $\begin{array}{c}802.11 \\
\text { channel }\end{array}$ & $\begin{array}{c}\text { ZigBee } \\
\text { channel }\end{array}$ & $\begin{array}{c}\text { I-factor based on } \\
\text { spectrum analyzer traces }\end{array}$ \\
\hline 3 & 11 & 0 \\
3 & 12 & 0.01 \\
3 & 13 & 0.84 \\
3 & 14 & 0.92 \\
3 & 15 & 0.96 \\
3 & 16 & 0.55 \\
3 & 17 & 0.02 \\
\hline
\end{tabular}

The ZigBee radio is the interferer to the 802.11 radio on channel 3 .

We generate 802.11 traffic by ping flooding. During the experiment, we collect spectrum analyzer traces (see Figures 14, 15, 16, and 17, where the receiver is an 802.11 radio operating on channel 3 (shown in blue) and the interferer radio is a ZigBee radio operating on channels 12 through 17 (shown in red)).

Table 2 summarizes the I-factor calculations using SIAM for these experiments. The first column shows that the 802.11 receiver is fixed at channel 3. The second column shows the 802.15.4 channel of the ZigBee interferer. The last column shows the I-factor values computed with our method. When the channel center frequencies of the 802.15 .4 and 802.11 radios are separated by at least 17 $\mathrm{MHz}$, the interference power that leaks from the 802.15.4 radio on the 802.11 receiver is 0 . If the center frequencies are separated by at least $12 \mathrm{MHz}$, the interference power from the 802.15.4 radio is still negligible. However, if the center frequencies are separated by less than $12 \mathrm{MHz}$, there is significant power leakage from the 802.15.4 radio.

\section{Conclusion}

In this article, we have proposed a novel measurementbased approach for computing the interference factor between two wireless radio channels. Our approach is generic because it is capable of computing the I-factor between channels of different wireless technologies, such as IEEE 802.11 and 802.15.4, as well as between channels of the same wireless technology. We have also proposed two specific measurement-based I-factor definitions and presented their computation methods following a measurement-based approach.

In our wireless testbed, we performed experiments with 802.11b and 802.15.4 radios and computed I-factor values between 802.11 channels and between 802.15.4 and 802.11 channels. We compared our results with the existing results in the literature and we reported new results on ZigBee to Wi-Fi interference. According to our findings, an 802.15.4 radio does not interfere with an $802.11 \mathrm{~b}$ receiver if their center frequencies are separated by at least $17 \mathrm{MHz}$. If the center frequencies are separated by $12 \mathrm{MHz}$, the interference from the 802.15.4 radio on the 802.11 receiver is still negligible. However, if the center frequencies are closer than $12 \mathrm{MHz}$, the interference from the 802.15.4 radio becomes significant in terms of the 802.15.4 radio's total power.

\section{Competing interests}

The authors declare that they have no competing interests.

\section{Acknowledgements}

This study was supported in part by European Union FP7 Programme with project FIRESENSE, FP7-ENV-2009-1-244088-FIRESENSE.

\section{Author details}

${ }^{1}$ Department of Computer Engineering, Bilkent University, 06800, Ankara, Turkey. ${ }^{2}$ Department of Electrical and Electronics Engineering, Bilkent University, 06800, Ankara, Turkey.

Received: 8 October 2012 Accepted: 7 February 2013

Published: 14 March 2013

\section{References}

1. A Mishra, V Shrivastava, S Banerjee, W Arbaugh, Partially overlapped channels not considered harmful. ACM SIGMETRICS Perform. Eval. Rev. 34(1), 63 (2006). [Online]. Available: [http://portal.acm.org/citation.cfm? doid $=1140103.1140286]$

2. IEEE Std 802.11-2007, IEEE Standard for Information Technology-Telecommunications and information exchange between systems-LANs and MANs-Specific requirements-Part 11: WLAN MAC and PHY Specifications (2007)

3. A Mishra, E Rozner, S Banerjee, W Arbaugh, in Proceedings of the 5th ACM SIGCOMM conference on Internet Measurement. Exploiting partially overlapping channels in wireless networks: turning a peril into an advantage (USENIX Association, 2005), pp. 29-29. [Online]. Available: [http://portal.acm.org/citation.cfm?id=1251115]

4. H Zimmermann, Open systems interconnection. IEEE Trans. Commun. 28(4), 425-432 (1980). [Online]. Available: [http://ieeexplore.ieee.org/xpls/ abs_all.jsp?arnumber=1094702]

5. TS Rappaport, Wireless Communications Principle and Practice, 2nd edn. (Prentice Hall Ptr, New Jersey, 2002)

6. Z Feng, Y Yang, in Proceedings of IEEE Wireless Communications and Networking Conference, WCNC'08. How much improvement can we get from partially overlapped channels? (2008), pp. 2957-2962. [Online]. Available: [http://ieeexplore.ieee.org/xpls/abs_all.jsp?arnumber=4489548]

7. Z Feng, Y Yang, in Proceedings of IEEE Global Telecommunications Conference, GLOBECOM'08. Characterizing the impact of partially overlapped channels on the performance of wireless networks, (2008), pp. 1-6. [Online]. Available: [http://ieeexplore.ieee.org/xpls/abs_all.jsp? arnumber $=4698733$ ]

8. G Zhou, JA Stankovic, SH Son, in Third IEEE Workshop on Embedded Networked Sensors, EmNets'06. Crowded spectrum in wireless sensor networks, (2006). [Online]. Available: [http://www.cs.virginia.edu/wsn/ docs/papers/emnets06-crowded.pdf]

9. P Fuxjäger, D Valerio, F Ricciato, in Proceedings of Fourth Annual IEEE Conference on Wireless on Demand Network Systems and Services, WONS'07. The myth of non-overlapping channels: interference measurements in IEEE 802.11, (2007), pp. 1-8. [Online]. Available: http://ieeexplore.ieee.org/ xpls/abs_all.jsp?arnumber $=4142700$

10. Y Yao, AUH Sheikh, S Cheng, in Proceedings of IEEE Pacific Rim Conference on Communications, Computers and Signal Processing. Near/far effects on packet radio networks with direct-sequence spread-spectrum signaling, (1989), pp. 122-125. [Online]. Available: [http://ieeexplore.ieee.org/xpls/ abs_all.jsp?arnumber $=48320]$

11. M Petrova, L Wu, P Mähönen, J Riihijärvi, in Proceedings of the Sixth International IEEE Conference on Networking, ICN'07. Interference measurements on performance degradation between colocated IEEE 802.11 g/n and IEEE 802.15.4 networks, (2007), pp. 93-98. [Online]. Available: [http://ieeexplore.ieee.org/xpls/abs_all.jsp?arnumber=4196286] 
12. Yellowjacket-Tablet Wi-Fi Analyzer (2012). available from: [http://www. bvsystems.com/Products/WLAN/YJ-TABLET/yj-tablet.htm], Last Access. [Online]. Available: [http://www.bvsystems.com/Products/WLAN/YJTABLET/yj-tablet.htm]

13. Maxim MAX2820, MAX2820A, MAX2821, MAX2821A 2.4GHz 802.11b Zero-IF Transceivers Data Sheet (2012). available from: [http://datasheets, maxim-ic.com/en/ds/MAX2820-MAX2821A.pdf], Last Access, [Online].

Available: [http://datasheets.maxim-ic.com/en/ds/MAX2820-MAX2821A. pdf]

14. Memsic Wireless Modules Product Page (2012). available from: [http:// www.memsic.com/wireless-sensor-networks/], Last Access, [Online]. Available: [http://www.memsic.com/wireless-sensor-networks/]

15. IEEE Std 802.15.4-2006, IEEE Standard for Information Technology-Telecommunications and information exchange between systems- Local and metropolitan area networks-Specific requirements-Part 15.4: Wireless Medium Access Control (MAC) and Physical Layer (PHY) Specifications for Low-Rate Wireless Personal Area Networks (WPANs) (2006)

doi:10.1186/1687-1499-2013-68

Cite this article as: Ulucinar et al: A novel measurement-based approach for modeling and computing interference factors for wireless channels. EURASIP Journal on Wireless Communications and Networking 2013 2013:68.

\section{Submit your manuscript to a SpringerOpen ${ }^{\circ}$ journal and benefit from:}

- Convenient online submission

Rigorous peer review

- Immediate publication on acceptance

- Open access: articles freely available online

- High visibility within the field

- Retaining the copyright to your article

Submit your next manuscript at $>$ springeropen.com 